\title{
The first description of the male of Cyclosa alba considering the partial sequence of mitochondrial CO1 gene
}

\author{
Akio Tanikawa ${ }^{1}$, Yusuke Ikeda ${ }^{2} \&$ Masanobu Yoshio ${ }^{1}$ \\ ${ }^{1}$ Laboratory of Biodiversity Science, School of Agriculture and Life Sciences, The University of Tokyo, 1-1-1, Yayoi, \\ Bunkyo-ku, Tokyo, 113-8657 Japan \\ E-mail:dp7a-tnkw@j.asahi-net.or.jp,yoshio@es.a.u-tokyo.ac.jp \\ 23-124-2, Onji-minamimachi, Yao-shi, Osaka, 581-0884 Japan \\ E-mail: yu_ikeda2739@snow.ocn.ne.jp
}

\begin{abstract}
The male of Cyclosa alba is described. Partial sequences of mitochondrial CO1 gene are used for species determination of the male. The candidate male specimen has the same haplotype as females of C. alba and nested in a strongly supported molophyletic clade of C. alba.
\end{abstract}

Key words - Taxonomy, spider, DNA barcoding, Japan

\section{Introduction}

The male of Cyclosa alba Tanikawa 1992 has been unknown since its original description. We found several unidentified male specimens of Cyclosa, which may be the candidate for C. alba. Because the emboli of palps of these specimens are filiform and wrapped in conductor similar to those of C. maritima Tanikawa 1992, C. mulmeinensis (Thorell 1887), C. sachikoae Tanikawa 1992, and C. vallata Keyserling 1886, the males in question seem to be related to those species. Females of $C$. alba also seem to be related to those species because their epigyna are prolate and have non-wrinkled scapes. These circumstantial evidence suggests that the males in question could be the male of $C$. alba. To confirm this hypothesis, we conducted a phylogenetic analysis using the partial sequences of mitochondrial $\mathrm{CO} 1$ gene of one candidate male and individuals of the above species group.

The voucher specimens of this paper are deposited in the collection of the department of zoology, National Museum of Nature and Science, Tokyo (NSMT). Nucleotide sequence data analyzed in this paper are available in the DDBJ/EMBL/GenBank databases.

We wish to express our heartfelt thanks to Dr. Tadashi Miyashita, the University of Tokyo, for his critical reading of the manuscript of this paper. We are deeply indepted to Dr. Yuki G. Baba, Dr. Mayura D. Takada, and Dr. Kentaro Uchiyama, the University of Tokyo, Dr. Yoh Ihara, and Ms. Naoko Hori, Hiroshima, Ms. Kazuyo Funabiki, Hyogo, Mr. Takuma Hatsushiba, and Mr. Shingo Hatsusiba, Tokyo, Mr. Ken-ichi Kumada, Mie, Mr. Koichi Nojima, Osaka, and Mr. Akihiko Yawata, Tokyo, for their kind help and offering specimens used in this study.

\section{Materials and methods}

Specimens analysed. The specimens used for the analysis were 6 ㅇ and 1 juvenile of Cyclosa alba Tanikawa 1992, 3 우 of C. maritima Tanikawa 1992, 4 우 of C. mulmeinensis (Thorell 1887), 4 우 of C. sachikoae Tanikawa 1992, and 3 우 of C. vallata Keyserling 1886, and the male in question collected from Hadano-shi, Kanagawa Pref. The sampling data are shown in the appendix.

DNA extraction, polymerase chain reaction and sequencing. Specimens were preserved in $99.5 \%$ ethanol, and genomic DNA was extracted from muscle of legs using DNeasy Blood \& Tissue kit (Qiagen, Inc.). The mitochondrial cytochrome oxidase I (mt-CO1) partial sequences were amplified using the primer combination LCOI-1498: 5'-GGT CAA CAA ATC ATA AAG ATA TTG G-3' with HCOI-2198: 5'-TAA ACT TCA GGG TGA CCA AAA AAT CA-3' (Folmer et al. 1994). The reactants were initially denatured for $2 \mathrm{~min}$ at $95^{\circ} \mathrm{C}$, proceeded with $40 \mathrm{cy}$ cles of $30 \mathrm{sec}$ at $95^{\circ} \mathrm{C}, 30 \mathrm{sec}$ at $47^{\circ} \mathrm{C}, 60 \mathrm{sec}$ at $72^{\circ} \mathrm{C}$ and then the final extension at $72^{\circ} \mathrm{C}$ for $5 \mathrm{~min}$. PCR products were purified using the ExoSAP-IT (GE Healthcare BioSciences, Co. Ltd.). The purified PCR products were sequenced using the BigDye terminator cycle sequencing kit and analysed on ABI 3100 automated DNA sequencer (Applied Biosystems, Foster City, CA). Chromatograms were checked by eye. Sequence alignments were done by Clustal W program (Thompson et al. 1994) in BioEdit version 7.0.5.3 (Hall 1999).

Phylogenetic analysis. The phylogenetic relationship was estimated by Neighbor Joining method, Bayesian inference, and Maximum Likelihood method. Neighbor Joining method was performed using MEGA ver.4.0 (Tamura et al. 2007). Substitution model was set as Maximum Composite 


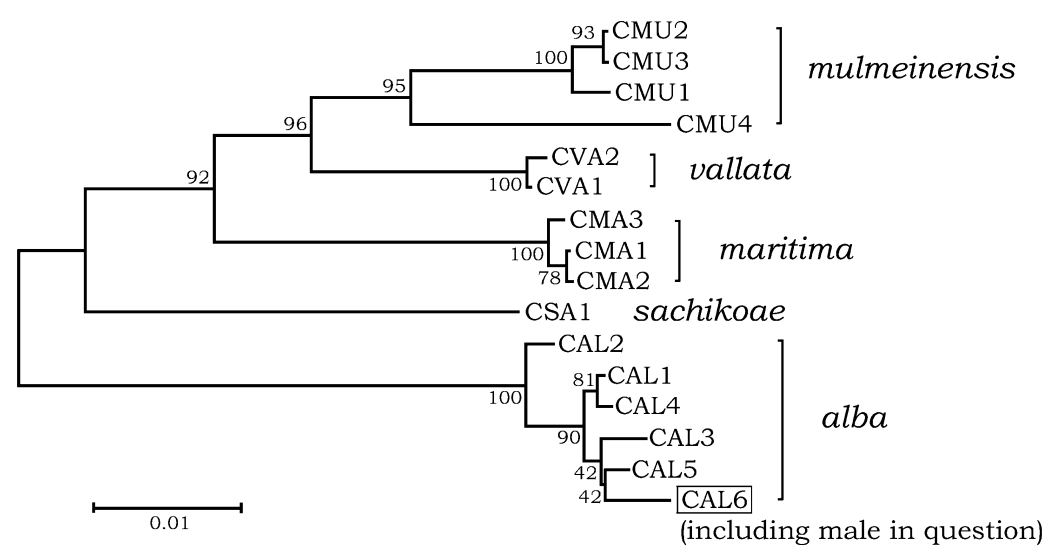

Fig. 1. Unrooted bootstrap consensus tree obtained by Neighbor Joining method for the species examined. Scores at each node are bootstrap values (1000 replicates). Scalebar shows substitution per site.

Likelihood and conducted 1000 replicates of bootstrapping test. The perl script MODELSELECT written by Akifumi Tanabe (available at http://www.fifthdimension.jp/) and PAUP ver. 4.0b10 (Swofford 2002) were used to determine the appropriate model of DNA evolution by AIC, AICc, and BIC for Bayesian inference. MrBays ver. 3.1.2 (Ronquist \& Huelsenbeck 2003) was employed to conduct Bayesian inference. Four concurrent Markov Chain Monte Carlo (MCMC) chains were run for 500,000 generations, saving a tree every 100 generations. Topologies prior to $\ln$ stabilization ("burn-in") were discarded and posterior clade probabilities were computed from the remaining trees. Maximum Likelihood method was performed using PHYML ver. 2.4.4 (Guindon \& Gascuel 2003) and conducted 1000 replicates of bootstrapping test.

\section{Results and conclusion}

We finally obtained $592 \mathrm{bp}$ of mt-CO1 partial sequences from all the specimens used. We found 6 haplotypes (CAL1-6) in C. alba, 3 in C. maritima (CMA1-3), 4 in C. mulmeinensis (CMU1-4), 1 in C. sachikoae (CSA1), and 2 in C. vallata (CVA1-2). The accession numbers of DDBJ/ EMSL/GenBank of those are shown in the appendix. The male in question has the same haplotype (CAL6) as two females of C. alba.

The best-fit model of sequence evolution determined by MODELSELECT using AIC was GTR + SS, and those using AICc and BIC were HKY + SS. GTR + SS and HKY + SS models could not be used in PHYML, so GTR $+\mathrm{I}+\mathrm{G}$ and $\mathrm{HKY}+\mathrm{I}+\mathrm{G}$ models were used. Phylogenetic trees obtained from Neighbor Joining method, Bayesian inferences using GTR + SS model and HKY + SS model, and Maximum Likelihood methods using GTR $+\mathrm{I}+\mathrm{G}$ model and $\mathrm{HKY}+\mathrm{I}+\mathrm{G}$ model were almost congruent. The unrooted bootstrap consensus tree inferred from 1000 bootstrap replicates of Neighbor Joining method is shown in Fig. 1. Slight differences were found in the intraspecific relationships of C. maritima and C. alba among 5 trees otained. CMA2 and CMA3 are united in the result of Maximam Likelihood method using GTR $+\mathrm{I}+\mathrm{G}$ model. The relationship among CAL3, 5, and 6 were unsolved in two Bayesian inferences. Nevertheless, all haplotypes of C. alba are united in one strongly supported molophyletic clade (bootstrap value or posterior probability: 100) in all trees obtained including the male in question. Consequently, we conclude that it is a male of C. alba.

\section{Description}

Cyclosa alba Tanikawa 1992

[Japanese name: Shiro-gomi-gumo]

(Figs. 2-4)

Cyclosa alba Tanikawa 1992, p. 62, figs. 182-189 [female holotype from Iriomotejima Is. (NSMT-Ar 2413), examined].

Specimens examined. The following male specimens were determined as the males of $C$. alba by comparing the palpal morphology, especially bifurcated paramedian apophysis, with that of the male used for the molecular work (NSMT-Ar 8102). 1ð, Yokozawa, Akiruno-shi, Tokyo, 12-VII-1992, A. Tanikawa leg. (NSMT-Ar 8101); 10, Mt. Koboyama, Hadano-shi, Kanagawa Pref., 10-VII2005, A. Yawata leg. (NSMT-Ar 8102, palp only, when alive, as in Fig. 2); 10 ${ }^{7}$ Ima-ura, Toba-shi, Mie Pref., 17- $\mathrm{X}^{-}$ 1999, K. Kumada leg. (NSMT-Ar 8103); 10 , Osaka Castle Park, Chuo-ku, Osaka-shi, Osaka Pref., 9-VIII-2006, N. Koike leg.; 1ऽ, Mt. Ryu-o-zan, Ibaraki-shi, Osaka Pref., 23VI-2007, Y. Ikeda leg.; 10 Hyogo Pref., 19-VII-2005, K. Nojima leg. (NSMT-Ar 8104); 10 ${ }^{\top}$, Kuritani-cho, Otake-shi, Hiroshima Pref., $22^{-} \mathrm{V}^{-}$ 1989, Y. Ihara leg. (NSMT-Ar 8105); 10, Takarajima Is., Toshima-mura, Kagoshima Pref., 25-III-2001, Y. G. Baba leg.; 1ð , Oku, Okinawajima Is., Okinawa Pref., 29-III2001, A. Tanikawa leg. (NSMT-Ar 8106).

Description of male. Coloration and markings. Carapace light brown to blackish brown. Abdomen white or dark brown, with dark colored marking (Fig. 2).

Measurements (in mm). Based on $1 \sigma^{\Uparrow}$ from Tokyo 


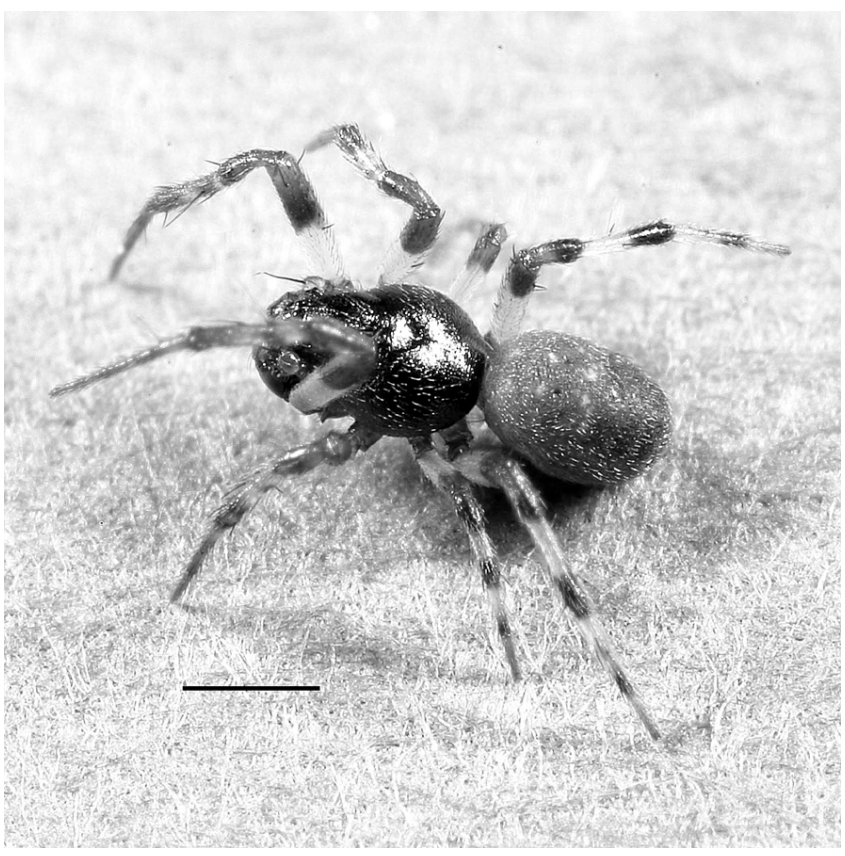

Fig. 2. Male of Cyclosa alba used for sequencing analysis, when alive (NSMT-Ar 8102). Scale: $1 \mathrm{~mm}$.
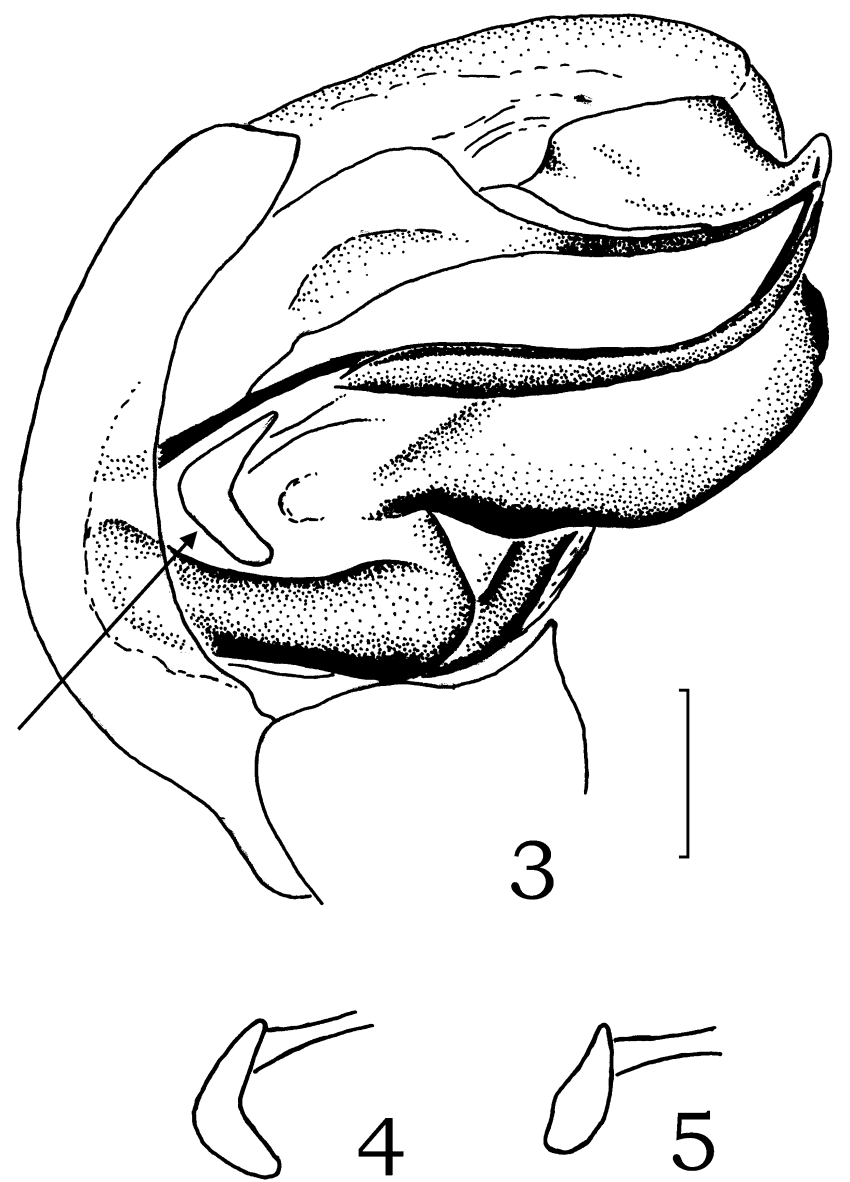

Figs. 3-5. 3-4, Cyclosa alba Tanikawa 1992 (NSMT-Ar 8102); 5, C. sachikoae Tanikawa 1992. _— 3, male palp, prolateral view, an arrow shows paramedian apophysis; 4-5, paramedian apophysis. Scale: $0.1 \mathrm{~mm}$.
(NSMT-Ar 8101), measurements in parentheses indicate the trait range of specimens examined. Body $2.76(2.36-2.77)$ long. Carapace $1.30(1.21-1.30)$ long, $1.13(0.98-1.13)$ wide. Length of legs [tarsus + metatarsus + tibia + patella + femur $=$ total]: I, $0.38+0.64+0.74+0.43+1.03=3.22 ; \mathrm{II}$, $0.36+0.58+0.56+0.43+0.98=2.91 ; \quad$ III, $0.35+0.38+$ $0.35+0.31+0.66=2.05 ; \quad$ IV,$\quad 0.35+0.65+0.68+0.39+$ $1.04=3.11$. Abdomen $1.52\left(1.18^{-1.52)}\right.$ long; $1.02\left(0.81^{-}\right.$ $1.28)$ wide.

Carapace longer than wide [length/width 1.15 (1.151.25)]. Median ocular area almost as long as wide [length/ width $0.96\left(0.93^{-1.04)}\right)$; wider in front than behind [anterior width/posterior width $1.36(1.27-1.57)]$. Labium wider than long [length/width $0.45\left(0.45^{-0.93)}\right)$. Sternum slightly longer than wide [length/width 1.04 (1.04-1.17)]. Length of leg I/length of carapace 2.48 (2.37-2.52). Abdomen longer than wide [length/width $1.49(1.13-1.49)$ ], anteriorly with a pair of dorsal tubercles, sometimes indistinct; and with a protuberance at the posterior end. Palp: embolus filiform, wrapped in an edge of conductor (Fig. 3); paramedian apophysis visible in prolateral view (Fig. 3 arrow) and distally bifurcated (Figs. 3, 4).

Remarks. The male of Cyclosa alba closely resembles $C$. sachikoae in general appearance and palpal morphology, but can be distinguished from the latter by the shape of paramedian apophysis. In alba, paramedian apophysis distally bifurcated (Figs. 3, 4), but not in sachikoae (Fig. 5).

Distribution. Japan (Honshu, Shikoku, Kyushu, Nansei Isls.).

\section{References}

Folmer, O., Black, M., Hoew, W., Lutz, R. \& Vrijenhoek, R. 1994. DNA primers for ampliation of mitochondrial cytochrome oxidase subunit I from diverse metazoan invertebrates. Mol. Mar. Biol. Biotechnol., 3: 294-299.

Guindon S. \& Gascuel O. 2003. A simple, fast, and accurate algorithm to estimate large phylogenies by maximum likelihood. Systematic Biology, 52: 696-704.

Hall, T. A. 1999. BioEdit: a user-friendly biological sequence alignment editor and analysis program for Windows 95/98/NT. Nucl. Acids Symp., 41: 95-98.

Ronquist, F. \& Huelsenbeck, J. P. 2003. MrBayes 3: Bayesian phylogenetic inference under mixed models. Bioinformatics, 19: 1572-1574.

Swofford, D. L. 2002. PAUP*. Phylogenetic analysis using parsimony (*and other methods), version 4.0b10. Sinauer Associates, Sunderland

Tamura, K., Dudley, J., Nei, M. \& Kumar, S. 2007. MEGA4: Molecular Evolutionary Genetics Analysis (MEGA) software version 4.0. Molecular Biology and Evolution 24: 1596-1599. (Publication PDF at http://www.kumarlab.net/publications)

Tanikawa, A. 1992. A revisional study of the Japanese spiders of the genus Cyclosa Menge (Araneae: Araneidae). Acta Arachnol., 41: $11-85$.

Thompson, J. D., Higgins, D. G. \& Gibson, J. 1994. Clustal W: improving the sensitivity of progressive multiple sequence alignment through sequence weighting, position- specific gap penalties and weight matrix choice. Nucl. Acids Res., 22: 4673-4680.

Received August 20, 2008 / Accepted September 30, 2008 
Appendix 1. Collection data, haplotype, DDBJ/EMSL/GenBank accession numbers of Cyclosa spiders used for DNA analysis.

\begin{tabular}{|c|c|c|c|c|c|}
\hline Species & Sex & Collecting sites & Date & Haplotype & Accession No. \\
\hline Male in question & $0^{\pi}$ & Hadano-shi, Kanagawa & $10-$ VII-2005 & CAL6 & AB453395 \\
\hline alba & 우 & Toyo-oka-shi, Hyogo & $25-$ VIII-2005 & CAL1 & AB453390 \\
\hline alba & 우 & Himeji-shi, Hyogo & $3-V-2007$ & CAL2 & AB453391 \\
\hline alba & 우 & Yokohama-shi, Kanagawa & $30-$ VIII-2005 & CAL3 & AB453392 \\
\hline alba & 우 & Iriomotejima Is., Okinawa & 20-IV-2005 & CAL4 & AB453393 \\
\hline alba & 우 & Kamogawa-shi, Chiba & 30-IX-2005 & CAL5 & AB453394 \\
\hline alba & juvenile & Yokohama-shi, Kanagawa & $14-X-2007$ & CAL6 & AB453395 \\
\hline alba & 우 & Yokohama-shi, Kanawaga & $30-$ VIII-2005 & CAL6 & AB453395 \\
\hline maritima & 우 & Mukojima Is., Ogasawara & 9-IV-2008 & CMA1 & AB453763 \\
\hline maritime & 우 & Otouto Is., Ogasawara & $14-I V-2008$ & CMA2 & AB453764 \\
\hline maritima & 우 & Hahajima Is., Ogasawara & 18-IV-2008 & CMA3 & AB453765 \\
\hline mulmeinensis & 우 & Iriomotejima Is., Okinawa & 21-IV-2005 & CMU1 & AB453766 \\
\hline mulmeinensis & 우 & Iriomotejima Is., Okinawa & 21-IV-2005 & CMU2 & AB453767 \\
\hline mulmeinensis & 우 & Yonagunijima Is., Okinawa & $3-\mathrm{VI}-2007$ & CMU3 & AB453768 \\
\hline mulmeinensis & 우 & Yonagunijima Is., Okinawa & $3-\mathrm{VI}-2007$ & CMU4 & AB453769 \\
\hline sachikoae & 우 & Iriomotejima Is., Okinawa & 22-IV-2005 & CSA1 & AB453770 \\
\hline sachikoae & 우 & Iriomotejima Is., Okinawa & 22-IV-2005 & CSA1 & AB453770 \\
\hline sachikoae & 우 & Iriomotejima Is., Okinawa & $6-\mathrm{VI}-2007$ & CSA1 & AB453770 \\
\hline sachikoae & 우 & Iriomotejima Is., Okinawa & $18-\mathrm{VI}-2008$ & CSA1 & AB453770 \\
\hline vallata & 우 & Fujisawa-shi, Kanagawa & $17-$ VIII-2005 & CVA1 & AB453771 \\
\hline vallata & 우 & Fujisawa-shi, Kanagawa & $17-$ VIII-2005 & CVA2 & AB453772 \\
\hline vallata & 우 & Oda-shi, Shimane & 21-VII-2006 & CVA2 & AB453772 \\
\hline
\end{tabular}

